\title{
Suppression of Bcl-2 by Antisense Oligonucleotides Results in Compensation and Enhanced Tumor Proliferation Involving KI-67
}

\author{
Marvin Rubenstein ${ }^{1,2,3,4 *}$, Courtney MP Hollowell ${ }^{2}$ and Patrick Guinan ${ }^{1,2,4,5}$ \\ ${ }^{1}$ Division of Cellular Biology, Hektoen Institute for Medical Research \\ ${ }^{2}$ Division of Urology, Stroger Hospital of Cook County \\ Departments of ${ }^{3}$ Biochemistry and ${ }^{4}$ Urology, Rush University Medical Center \\ ${ }^{5}$ Department of Urology, University of Illinois at Chicago, Chicago, IL, 60612
}

Received: 17 June, 2015; Accepted: 25 August, 2015; Published: 20 September, 2015

*Corresponding author: Dr. Marvin Rubenstein, Chairman-Division of Cellular Biology, Hektoen Institute for Medical Research, 2240 West Ogden Avenue, 2'nd floor, Chicago, IL 60612, USA, Tel: 312-864-4621; Fax: 312-768-6010; E-mail: DrMarv@Prodigy.net

\begin{abstract}
Antisense oligonucleotides (oligos) have targeted regulatory proteins in both in vivo and in vitro cancer models including those of the prostate. In efforts to identify compensatory alterations in the expression of non-targeted genes this study evaluated monoand bispecific oligos which targeted and equally suppressed the expression of Bcl-2 (an apoptosis inhibitory protein). Previous studies have shown that treated LNCaP cells compensate for diminished Bcl-2 by suppressing caspase-3 (an apoptosis promoter) while enhancing the expression of AKT-1 (another apoptosis inhibitor), the Androgen Receptor (AR) and its p300 and IL-6 coactivators. Additional proteins enhanced include PD-1, its ligand PD-L1 (immune checkpoint blockade markers) and fas-ligand, which activate apoptosis through signal transduction, along with suppressor protein p53, oncogene v-myc, polymerase transcription mediator MED-12 and signal transducer STAT-3. This suggests that therapeutic approaches to restore apoptosis through suppression of bcl-2 leads to altered expression of non-targeted genes not only involving apoptosis, but also androgen sensitivity, transcriptional activity (including that of suppressor/ oncogenes) and immune responsiveness.
\end{abstract}

To further evaluate adaptive, compensatory mechanisms related to tumor resistance we now evaluate the expression of the proliferation antigen KI-67. We find the expression of this tumor proliferation marker greatly enhanced, particularly when compared to other previously reported non-targeted proteins, and propose that oligo-mediated treatment could be more effective when directed towards KI-67 rather than bcl-2. This could be accomplished by a monospecific oligo targeting KI-67, or with a bispecific simultaneously targeting bcl-2 and KI-67.

Keywords: Antisense; BCL-2; Prostate cancer; Therapy

\section{Introduction}

Gene therapy is, in theory, specific but encounters difficulties in practice. While suitable targets are found in many pathways, and tumors express altered patterns of gene expression, the actual activity of most regulatory genes is similar to normal cells. Resistance develops because the biochemical pathways involved are complex and regulated by combinations of both stimulatory and inhibitory factors; many affected by therapy, and some of these affected genes are susceptible to altered patterns of expression having the potential to reverse the effects of the intended treatment. Our studies have shown that the evaluation of non-targeted genes, following what was believed to be specific gene therapy, suggests that tumors frequently alter their dependence on the initially targeted gene products, by relying upon others through the process of compensation [1]. Just as bacteria and viruses mutate to evade antibiotic and antiviral agents, tumor cells are under similar selective pressure to evade therapy, whether chemical (chemotherapy), hormonal (deprivation), radiologically induced, or that based on suppression of gene translation (including the use of antisense oligonucleotides [oligos]). While oligo based therapy is already in clinical trials, the unintended compensatory consequences of intervention are poorly understood, and could contribute to the emergence and selection of more aggressive tumor cells.

Gene therapy has been clinically employed for the treatment of human prostate tumors and, employed with either radio therapy [2,3] or chemotherapy [4], antisense oligos have been administered against inhibitors of apoptosis (particularly bcl-2 and clusterin) in attempts to restore that process mediated by the mitochondrial pathway. In addition, a small bioavailable molecular inhibitor of bcl-2 (venetoclax [GDC-0199/ABT-199]) is currently being evaluated in Phase I and II studies by Genentech for the treatment of a variety of tumor types. Additional targets to regulate apoptosis could also include those involved with activation mediated by signal transduction and initiated via cell surface receptors and their respective ligands (PD-1/PD-L1 and fas/fas-ligand). If such therapy is to be successful, it is important 
to examine mechanisms by which tumors compensate and become resistant.

We previouslyreported that LNCaPcells treated with antisense oligos directed against bcl-2 (administered in a nanoparticle suspension of lipofectin) compensated by suppressing caspase-3 [1] (an apoptosis promoter), and enhancing AKT-1(an inhibitor of apoptosis) [5], androgen receptor [6] (AR), co-activating p300 [7] and IL-6 expression [8]. In addition PD-1, its ligand PD-L1 (immune checkpoint blockade markers) and fas-ligand, which activate apoptosis through signal transduction, were also enhanced [9], as were suppressor protein p53 [10], oncogene v-myc [11], polymerase transcription mediator MED-12 [12] and signal transducer STAT 3 [5,12]. This suggests that, in LNCaP, therapeutic approaches to restore apoptosis (including the use of antisense oligos) which suppress bcl-2 can lead to altered expression in non-targeted genes and regulatory changes not only involving apoptosis, but also androgen sensitivity, (suppressor/oncogene) transcriptional activity and immune responsiveness. Many of the androgen related gene expression alterations are similar to those associated with more advanced prostate tumors, while regulation of apoptosis involved both mitochondrial and signal transducing pathways. As noted above we recently demonstrated that cell surface proteins which regulate immune checkpoint blockades (PD-1 and PD-L1 and fasligand) and activate apoptosis via signal transduction were also enhanced [9], presumable leading to further inhibition of T-cell activity. Compensatory effects identified with these proteins are important since the PD-1/PD-L1 pathway is now recognized as a target for monoclonal antibody directed immunotherapy used to treat various solid tumors.

Previous studies mentioned in this report evaluated compensatory effects upon apoptosis, androgen regulation, angiogenesis, autocrine, (oncogene/suppressor) transcriptional proteins, immunologic recognition, antigen expression. We now evaluate the expression of the proliferation antigen KI-67 with the expectation that as a result of the numerous compensatory changes noted above, some of which suggesting emergence of a more aggressive tumor phenotype, increased proliferation should follow. If so, then KI-67, considered to be a highly regulated protein associated with proliferation status, could be greatly enhanced [13], and possibly could provide a better (or additional) therapeutic target for antisense oligos than bcl2. The biological significance for studying KI-67 is that it is a marker which is exclusively associated with proliferation, and its expression is considered a prognostic indicator for some tumors (breast). It is expressed throughout all phases of cell division $\left(G_{1}, S, G_{2}\right.$, and mitosis) but absent while cells rest $\left(G_{0}\right)$. Some consider it a therapeutic target and have employed antisense oligos against KI-67 demonstrating inhibition of both in vitro and in vivo growth [13] in various models. It also induced apoptosis and reversed tumorigenicity of breast cancer cells [14]. For gene therapy to ultimately be successful it must be made more specific and mechanisms of compensation must be identified and possibly targeted for additional suppression. This could include the use of mono-specific gene regulatory (oligos) agents employed simultaneously, but could also include either bispecific or even (proposed) multispecific oligo-based agents [15], which simultaneously target bcl-2 and KI-67.

We employed RT-PCR in these experiments to determine relative alterations in gene expression. Although more sophisticated techniques are available we find this method both sensitive enough to identify those genes involved with compensation, and capable of identifying non-targeted genes (like KI-67) which are particularly affected and could provide new targets for suppressive gene therapy.

\section{Methods}

\section{Oligonucleotides}

Oligos (mono- or bispecific) were purchased from Eurofins MWG Operon (Huntsville, AL). Each was phosphorothioated on three terminal bases at 5' and 3' positions. Stock solutions were made to a final concentration of $625 \mu \mathrm{M}$ in sterile Dulbecco PBS.

\section{Base sequences}

Each oligo contained at least one CAT sequence and targeted the area adjacent to the mRNA AUG initiation codon for the respective targeted protein (EGFR or bcl-2).

$\mathbf{M R}_{4}$ (monospecific targeting bcl-2) T-C-T-C-C-C-A-G-C-G-TG-C-G-C-C-A-T

$\mathbf{M R}_{24}$ (bispecific targeting EGFR/bcl-2) G-A-G-G-G-T-C-G-CA-T-C-G-C-T-G-C-T-C-T-C-T-C-C-C-A-G-C-G-T-G-C-G-C-C-A-T

$\mathbf{M R}_{42}$ (bispecific targeting bcl-2/EGFR) T-C-T-C-C-C-A-G-C-GT-G-C-G-C-C-A-T-G-A-G-G-G-T-C-G-C-A-T-C-G-C-T-G-C-T-C

\section{Cell culture}

LNCaP cells were grown in RPMI 1640 supplemented with $10 \%$ bovine serum, 1\% L-glutamine and 1\% penicillin/ streptomycin under a $5 \% \mathrm{CO}_{2}$ incubator. Log phase cells were harvested using EDTA/trypsin and equally distributed into $75 \mathrm{~cm}^{2}$ flasks (Corning, NY). At intervals, media were either supplemented or replaced with fresh.

\section{Oligo treatment prior to PCR}

Fours days prior to oligo addition, when cell density approached $75 \%$ confluence, $10 \mathrm{ml}$ of fresh media was added. Cells were incubated for an additional 3 days before $5 \mathrm{ml}$ of media was replaced with fresh the day before oligos were added. $100 \mu \mathrm{l}$ of stock oligos were added to bring the final concentration to $6.25 \mu \mathrm{M}$. Incubation proceeded for an additional 24 hours in the presence or absence of monospecific $\mathrm{MR}_{4}$, or the $\mathrm{MR}_{24}$ and $\mathrm{MR}_{42}$ bispecifics in a nanoparticle suspension with lipofectin.

\section{RNA extraction}

Following treatment, media was removed, a single ml of cold $\left(4^{\circ} \mathrm{C}\right)$ RNAzol B was added to each $75 \mathrm{~cm}^{2}$ culture flask and the monolayer lysed by repeated passage through a pipette. All procedures were performed at $4^{\circ} \mathrm{C}$. The lysate was removed, placed in a centrifuge tube to which $0.2 \mathrm{ml}$ of chloroform was added and shaken. The mixture stayed on ice for $5 \mathrm{~min}$, was spun at $12,000 \mathrm{~g}$ for $15 \mathrm{~min}$, and the upper aqueous volume removed 
and placed in a fresh tube. An equal volume of isopropanol was added, the tube shaken, and allowed to stay at $4^{\circ} \mathrm{C}$ for $15 \mathrm{~min}$ before similar centrifugation to pellet the RNA. The supernatant was removed, the pellet washed in a single $\mathrm{ml}$ of $75 \%$ ethanol, then spun for $8 \mathrm{~min}$ at $7500 \mathrm{~g}$. The ethanol was pipetted off and the formed pellet was air dried at $-20^{\circ} \mathrm{C}$.

\section{RNA quantitation}

RNA was resuspended in $250 \mu \mathrm{l}$ of DEPC treated $\mathrm{H}_{2} \mathrm{O}$ and quantitated using a Qubit fluorimeter and Quant-iT RNA assay kit (Invitrogen). DEPC is an inhibitor of RNase activity.

\section{RT-PCR}

Extracted RNA was diluted in DEPC treated water to 40 $\mu \mathrm{g} / \mu \mathrm{l}$. 1-4 $\mu \mathrm{l}$ of this RNA was added to $1 \mu \mathrm{l}$ of both sense and antisense primers (forward and reverse sequences) for actin, bcl-2, and KI-67. From a kit purchased from Invitrogen the following reactants were added for RT-PCR: $25 \mu \mathrm{l}$ of $2 \mathrm{X}$ reaction mixture, $2 \mu$ S SuperScript III RT / platinum Taq mix, tracking dye, and $3 \mu \mathrm{MgSO}_{4}$ (of a $5 \mathrm{mM}$ stock concentration). DEPC treated water was added to yield a final volume of $50 \mu \mathrm{l}$. RT-PCR was performed for 2 X 25 cycles using the F54 program in a Sprint PCR Thermocycler. As a control for RT-PCR product production, human actin expression was tested in RNA extracted from HeLa cells which was provided in a kit purchased from Invitrogen (in the reaction mixture, no $\mathrm{MgSO}_{4}$ was included, the difference was compensated for by $3 \mu \mathrm{l}$ of DEPC treated water).

\section{Primers}

\section{Actin :}

Forward primer sequence: 5' CAA ACA TGA TCT GGG TCA TCT TCT C 3

Reverse primer sequence: 5’ GCT CGT CGT CGA CAA CGG CTC PCR product produced was 353 base pairs in length

Bcl-2:

Forward primer sequence: 5' GAG ACA GCC AGG AGA AAT CA 3'

Reverse primer sequence: 5’ CCT GTG GAT GAC TGA GTA CC 3'

PCR product produced was 127 base pairs in length.

\section{KI-67:}

Forward primer sequence: 5' TTG GAG AAT GAC TCG TGA GC 3'

Reverse primer sequence: 5' CGA AGC TTT CAA TGA CAG GA 3'

PCR product produced was 218 base pairs in length.

\section{Detection and Quantitation of Product}

\section{Agarose gel electrophoresis}

$1.5 \%$ agarose gels were prepared in a $50 \mathrm{ml}$ volume of TBE buffer (1X solution: $0.089 \mathrm{M}$ Tris-borate and 0.002M EDTA, pH 8.3), containing $3 \mu \mathrm{l}$ of ethidium bromide in a Fisher Biotest electrophoresis system. Samples were run for 2 hours at a constant voltage of 70 using a Bio-Rad 1000/500 power supply source. To locate the amplified PCR product, $3 \mu \mathrm{l}$ of a molecular marker (Invitrogen) which contained a sequence of bases in 100 base pair increments (Invitrogen) as well as $2 \mu \mathrm{l}$ of a sucrose based Bromophenol blue tracking dye were run in each gel.

\section{Quantitation}

Gels were visualized under UV light and photographed using a Canon 800 digital camera. Photos were converted to black and white format and bands quantitated using Medical Image Processing, analysis and Visualization (Mipav) software provided by the National Institute of Health. Means and standard deviations were compared using Student $t$-tests to determine significance.

\section{Results}

\section{Bcl-2 expression}

As a control (Figure 1) for RT-PCR product production, human actin expression was tested in RNA extracted from HeLa cells [1].

LNCaP cells incubated for 24 hours in the presence of $6.25 \mu \mathrm{M}$ of oligos suppressed bcl-2 expression, and support the finding of comparable biologic activity in both mono- and bispecific oligos measured in the in vitro cell growth inhibition experiments [1]. When photographs of the identified product bands were scanned on agarose gels and quantitated using Mipav software, in a series of runs, the greatest expression of bcl-2 was always found in untreated LNCaP cells. Those treated with oligos, whether mono- or bispecific, produced bands which indicated obvious (to the naked eye) suppression. For each oligo evaluated, the

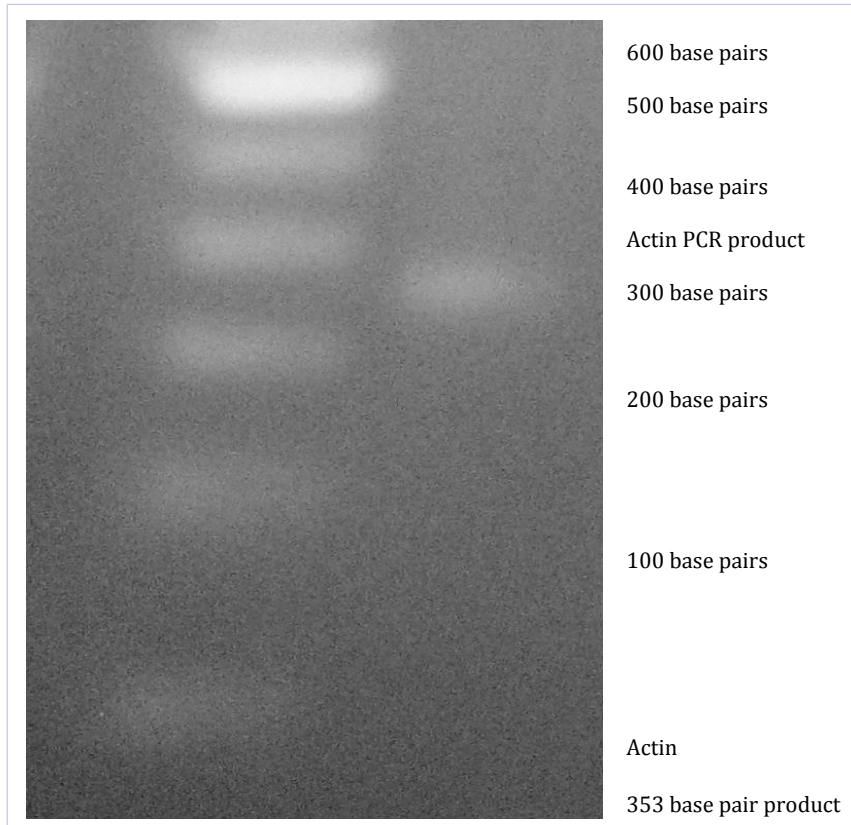

Figure 1: Human actin control in an agarose gel. 
greatest amount of suppression measured approached 100\% for the mono-specific $\mathrm{MR}_{4}$; and for the bispecific $\mathrm{MR}_{24}$ and $\mathrm{MR}_{42}$, with $86 \%$ and $100 \%$, respectively. Suppression was found in both repeat PCR runs with bcl-2 primers, as well as in repetitive agarose gel quantifications [16]. A representative band is presented in Figure 2.

\section{KI-67 expression}

Identical amounts of extracted RNA from LNCaP cells treated with either mono- or bispecific oligos directed against bcl-2 (and EGFR in the bispecifics) were then evaluated by RT-PCR using primers directed against KI-67. When background intensity was subtracted, the relative intensity of all bands corresponding to KI67 representing cells treated with $\mathrm{MR}_{4}, \mathrm{MR}_{24}$ and $\mathrm{MR}_{42}$ compared to controls were respectively increased $363.0 \% \pm 158.9(\mathrm{P}=$ $0.000229), 427.1 \% \pm 232.3(\mathrm{P}=0.001137)$ and $355.7 \% \pm 210.5$ $(\mathrm{P}=0.002017)$. These results were pooled from six duplicate gels and the statistically significant increased expression found in each type of oligo are among the greatest levels of relative increase seen in all previously reported experiments [1,5-7,812,15-18]. A representative gel is found in Figure 3.

\section{Discussion}

Gene therapy is often promoted as a highly specific and deliverable treatment to control aberrant gene expression by tumor cells (particularly when growth factors, their receptors or apoptosis inhibitors are excessively produced). However, it's now apparent that it's not as specific as previously thought. Antisense oligos consist of nucleotide bases, which synthesized complimentary in sequence to mRNA. When hybridized to mRNA, they produce a translational arrest of the targeted gene's mRNA expression. Now in clinical trials against a variety of solid tumors, this method is an effective, relatively non-toxic and inexpensive form of therapy and various types of antisense RNA have been constructed for this purpose. These include the

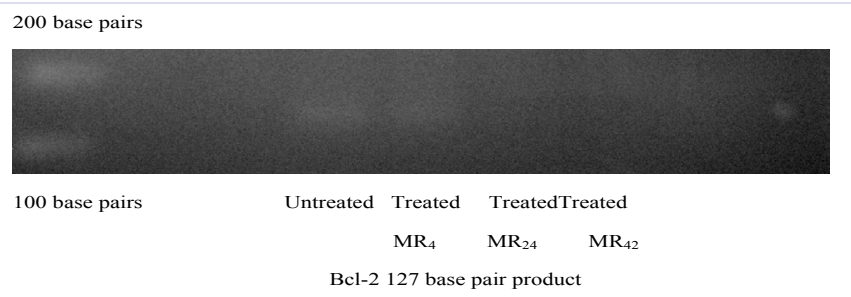

Figure 2: Bcl-2 expression is suppressed by oligos as indicated in a representative agarose gel.

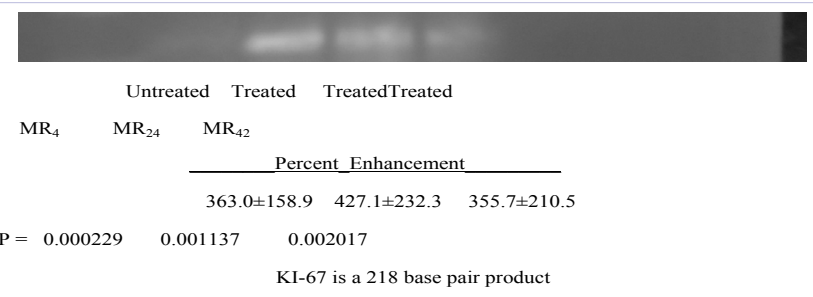

Figure 3: KI-67 expression is greatly enhanced by oligo treatment as indicated in a representative agarose gel. phosphorothioate oligos used in these evaluations and other formulation including 2'-MOE-RNA, morpholinos, siRNA, miRNA etc. Modifications to the oligo backbone and base structure are used to prevent nuclease degradation, increase systemic half- life, cross the blood-brain barrier or enhance distribution and delivery. Some of these derivatives have been evaluated clinically, but all are directed against single gene transcriptional (mRNA) products. In contrast, the oligos discussed in this paper included both mono- and bispecific forms, each having a base sequence complementary to and directed against mRNA encoding the apoptosis inhibitor bcl-2, (bispecifics included an additional site directed against Epidermal Growth Factor Receptor [EGFR]). We developed and evaluated bispecific oligos because it would be naïve to believe targeting a single mRNA would be sufficient to produce a clinical response in most tumors, and activity at one site does not affect binding at a second [16]. Therefore administration of a single oligo having two mRNA targets could have an additional suppressive effect, and we have shown that both types of oligos have comparable activity when suppressing bcl-2 [16].

While it's understandable that genes which share sequence homology would also be susceptible to antisense oligos, when directed at common sequences, what is not expected are the effects on non-targeted genes, many of which control additional growth regulating pathways. We have also shown that certain complementary stretches of base sequences within the oligo could also produce unanticipated effects on the expression of cell surface antigens (and differentiation proteins). In an early evaluation of bispecifics we reported the enhanced expression of Prostate Specific Membrane Antigen (PSMA) [17] when oligos were directed against bcl-2. The unique capacity to produce such changes by these bispecifics (and not a similarly directed monospecific) is attributable to an unusual double strand conformation present in bispecifics, due to this complementarity between intrastrand bases, which induces interferon (an enhancer of surface antigen expression) [18]. Such expression could enable better recognition and targets by cytotoxic $\mathrm{T}$ cells [18].

Tumors are a mass of genetically unstable heterogeneous cells capable of both rapid mutation and selection. Just as bacteria and viral agents develop resistance to chemical, hormonal and radiotherapy, tumors cells have a similar capability. In prostate cancer, it's thought that hundreds of genes (including those listed here) drive tumor cells to grow, in addition to the effects of androgen acting through the AR (as a transcriptional factor). AR coactivating proteins (p300, IL4, IL6), regulators of apoptosis (bcl-2, clusterin, AKT-1), additional transcriptional factors (MED12) and various autocrine loops (involving transforming growth factor-alpha [TGF- $\alpha$ ], its EGFR binding site, insulin like growth factor 1 [IGF1] and its receptor [IGF-1R]) contribute to both positive and negative regulation of tumor growth, and many (as we have seen) are affected by compensatory changes in their expression, following treatment (including oligo suppression of bcl-2). Initial evaluation of protein expression associated with compensation regulating the traditional mode of apoptosis 
(mitochondrial mediated) focused on the bcl-2, bax, bad, clusterin, proteins etc. However, more recent work evaluated proteins associated with tumor cell destruction, via apoptosis, mediated by a secondary route for activation, involving direct signal transduction. This is a process of initiating apoptosis through the binding of activating proteins (ligands) to cell surface receptors. When ligands bind to these receptors they activate a destructive cascade of protein interactions which lead to cell death. These receptors are structurally similar to the tumor necrosis factor receptor (CD95) and also regulate the immune system's cytotoxic T cell response. As mediators of the immune system their expression can have unanticipated effects on certain types of therapy since these proteins, particularly PD-1 and PDL1, are now being targeted by monoclonal antibodies to treat cancer patients via immune checkpoint blockade, particularly those with melanoma, mesothelioma and lung cancer [19]. Our studies found that PD-1 its ligand PD-L1 and fas-ligand, were all significantly enhanced following bcl-2 suppression and, therefore, contribute to an additional pathway for compensatory based resistance and control of apoptosis [9].

Oligos (produced by Oncogenex Pharmaceuticals) have reached clinical trials for the treatment of prostate cancer (OGX-011), while others remain in preclinical development (OGX-225). Often administered in combination with traditional chemotherapy, these oligos target bcl-2, clusterin (OGX-011 in Phase II testing), heat shock protein 27 (OGX-427) or Insulin growth factor binding proteins (OGX-225) [20]. Genta conducted a phase 3 test using oligos (Genasense; oblimersen) directed against bcl-2 for treating melanoma, chronic lymphocytic leukemia and various solid tumors [21], but compensatory effects produced by this agent were not reported. Many represent efforts to restore tumor apoptosis by eliminating suppressive bcl-2 [24] associated with treatment resistance. Similar approaches are directed at clusterin, but compensatory mechanisms activated by these oligos have not been evaluated. Since derivatives of antisense oligos (siRNA, miRNA) continued to developed and tested, and while some directed against bcl-2 are in clinical trials, it is important to identify compensatory changes that result.

This year (2015) the American Cancer Society (ACS) estimated that in spite of early detection, screening for Prostate Specific Antigen (PSA) and effective treatments for localized disease, in the United States there will be an estimated 27,540 deaths from prostate cancer with 220,800 newly diagnosed cases [22]. New types of treatment, including gene therapy and translational inhibition must be developed and employed (probably in combination with traditional androgen ablation).

\section{Conclusions}

These results demonstrate that one of the greatest changes in expression resulting from suppression of bcl-2 is the compensation involving the enhanced expression of the proliferation marker KI-67, which is associated with all phases of cell division. The statistically significant change we report here was one of the greatest found in all of our previous studies. This finding validates our previously reported results by identifying their cumulative effect upon proliferation, but also suggests KI-67 as a target for oligo based therapy, either alone or in combination with that directed towards bcl-2. Such therapy could be delivered by monospecific oligos separately targeting KI-67 and bcl-2, a bispecific oligo targeting both KI-67 and bcl-2, or a proposed [15] multispecific branched derivative targeting these and additional targets to be determined.

\section{Acknowledgments}

The Cellular Biology laboratory at the Hektoen Institute is supported, in part, by the Blum Kovler Foundation, the Cancer Federation, Safeway/Dominicks Campaign for Breast Cancer Awareness, Lawn Manor Beth Jacob Hebrew Congregation, the Max Goldenberg Foundation, the Pritzker Taubert Family Foundation, the Sternfeld Family Foundation, and the Herbert C. Wenske Foundation.

\section{References}

1. Rubenstein M, Hollowell CM, Guinan P. Inhibition of bcl-2 by antisense oligonucleotides is followed by a compensatory suppression of caspase-3 in LNCaP cells. In Vivo. 2011; 3: 1-6.

2. Mu Z, Hachem P, Pollack A. Antisense Bcl-2 sensitizes prostate cancer cells to radiation. Prostate. 2005 65: 331-340.

3. Yip KW, Mocanu JD, Au PY, Sleep GT, Huang D, Busson P, et al. Combination bcl-2 antisense and radiation therapy for nasopharyngeal cancer. Clin Cancer Res. 2005 11: 8131-8144.

1. 1.Yamanaka K, Miyake H, Zangemeister-wittke U, Jansen B and Gleave M. Novel bispecific antisense oligonucleotides inhibiting both Bcl-2 and Bcl-XL expression induce apoptosis and enhance chemosensitivity in human androgen-independent prostate cancer cells. Proceedings AACR 45. 2004

4. Rubenstein M, Hollowell CM, Guinan P. Additional compensatory mechanisms -altering antisense oligonucleotide suppression of Bcl-2: Effects upon AKT-1 and STAT.-3. In Vivo. 2014; 28: 867-870.

5. Rubenstein M, Hollowell CM, Guinan P. In LNCaP cells enhanced expression of the androgen receptor compensates for bcl-2 suppression by antisense oligonucleotides. Ther Adv Urol. 2011; 3: 73-79.

6. Rubenstein M, Hollowell CM, Guinan P. In LNCaP cells enhanced expression of both androgen receptor and co-stimulatory protein p300 compensate for antisense oligonucleotide suppression of bcl-2. Ther Adv Urol. 2011 3: 243-250.

7. Rubenstein M, Hollowell CM, Guinan P. Increased expression of the androgen receptor with p300 and IL- 6 coactivators compensate for oligonucleotide suppression of bcl-2. No increased CREBBP or IL-4 expression. Ther Adv Urol. 2013; 5: 85-93.

8. Rubenstein M, Hollowell CM, Guinan P. Following inhibition of BCL-2 by antisense oligonucleotides compensatory suppression of apoptosis involves the direct signal transduction pathway of LNCaP cell. Online Journal of Apoptosis. 2015; 4:1-10.

9. Rubenstein M, Hollowell CM, Guinan P. Oligonucleotide suppression of bcl-2 in LNCaP cells is compensated by increased androgen sensitivity, p53 and oncogene activity, and suppressed caspase- 3 . Med Oncol. 2013; 30: 599.

10. Rubenstein M, Hollowell CM, Guinan P. No compensation in VEGF expression follows antisense suppression of bcl-2 activity. In Vivo. 2012; 26: 937-940. 
11. Rubenstein $\mathrm{M}$, Hollowell $\mathrm{CM}$, Guinan $\mathrm{P}$. In LNCaP inhibition of bcl-2 by nucleotides results in compensatory changes involving apoptosis, transcription and immunoregulation. Enliven: J Genet Mol Cell Biol. 2014; $1: 001$

12. Kausch I, Lingnau A, Endl E, Sellmann K, Deinert I, Ratliff TL, et al Antisense treatment against Ki-67 mRNA inhibits proliferation and tumor growth in vitro and in vivo. Int J Cancer. 2003; 105: 710-716.

13. Wang R, Luo D, Ma X, Yang W, Chen R, Liu Y, et al. Antisense Ki-67 cDNA transfection reverses the tumorigenicity and induces apoptosis in human breast cancer cells. Cancer Invest. 2008; 26: 830-835

14. Rubenstein M, Anderson KM, Tsui P, Guinan P. Synthesis of branched antisense oligonucleotides having multiple specificities: Treatment of hormone insensitive prostate cancer. Med Hypotheses. 2006; 67: $1375-1380$

15. Rubenstein M, Guinan P. Bispecific antisense oligonucleotides have activity comparable to monospecifics in inhibiting expression of $\mathrm{Bcl}-2$ in LNCaP cells. In Vivo. 2010; 24: 489-493.
16. Rubenstein M, Hollowell CM, Guinan P. Increased prostate specific membrane antigen expression in LNCaP Cells following treatment with bispecific antisense oligonucleotides directed against bcl-2 and EGFR. Med Oncol. 2010; 27: 1212-1218.

17. Rubenstein M, Hollowell CM, Guinan P. Bispecific oligonucleotides may induce interferon in LNCaP cells enhancing surface antigen expression: Effect of intra strand base pair complementarity. In Vivo. 2011 25: 61-67.

18.http://www.washingtonpost.com/business/studies-merck-drugkeytruda-effective-against-3-cancers/2015/04/19/01d94cfc-e6b411e4-8581-633c536add4b_story.html

19. www.Oncogenex.ca

20. Kling J. Safety signal dampens reception for mipomersen antisense. Nat Biotechnol. 2010; 28: 295-297.

21.Siegal RL, Miller KD, Jemal A. Cancer Statistics, 2015. CA: A cancer journal for clinicians. 2015; 65: 5-29. 